\title{
Androgen receptor in hepatocarcinogenesis: Recent developments and perspectives (Review)
}

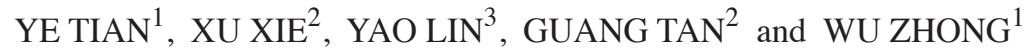 \\ ${ }^{1}$ Department of Emergency Medicine, Affiliated Hospital of Luzhou Medical College, Luzhou, Sichuan 646000; \\ ${ }^{2}$ Department of General Surgery, First Affiliated Hospital, Dalian Medical University, Dalian, Liaoning 116011; \\ ${ }^{3}$ Department of Urology, Anhui Provincial Hospital, Hefei, Anhui 230001, P.R. China
}

Received June 5, 2014; Accepted February 13, 2015

DOI: $10.3892 / \mathrm{ol} .2015 .3025$

\begin{abstract}
Previous studies have indicated that males are at a higher risk of developing hepatocellular carcinoma (HCC) compared with females. Identifying the factors that cause this gender-specific difference in the incidence of HCC has long been considered important for revealing the molecular mechanisms involved in hepatocarcinogenesis. Given the unprecedented tools that are now available for molecular research, genetic studies have established that the androgen receptor (AR) may be partly responsible for gender disparity in HCC. AR has a dual role, promoting HCC initiation and development, as well as suppressing HCC metastasis. The present review provides an overview of the involvement of AR signaling in HCC. The review highlighted important studies, examples of the direct AR transcriptional target genes involved in HCC and novel theories concerning the conventional concept, suggesting that targeting the AR, rather than the androgen, may provide an improved therapeutic approach for the treatment of HCC.
\end{abstract}

\section{Contents}

1. Introduction

2. AR alterations in $\mathrm{HCC}$

3. AR promotes hepatocarcinogenesis

4. AR suppresses HCC metastasis

5. AR as a promising target for cancer therapy

6. Conclusions

\section{Introduction}

Hepatocellular carcinoma (HCC) is the most common primary malignant tumor of the liver and, overall, one of the most

Correspondence to: Professor Wu Zhong, Department of Emergency Medicine, Affiliated Hospital of Luzhou Medical College, 25 Tai Ping Jie, Luzhou, Sichuan 646000, P.R. China E-mail: 15228471063@163.com

Key words: androgen receptor, hepatocellular carcinoma frequently diagnosed malignancies worldwide, accounting for $>1$ million annual mortalities (1). A large number of risk factors for HCC have been established, including hepatic viral infections, environmental exposure, alcohol consumption, genetic metabolic diseases, smoking, cirrhosis and oral contraceptives (2-4). Furthermore, comparative studies have identified that males are 2-8 times more likely to develop HCC compared with females in low- and high-incidence areas (5-7).

Previous studies support that the androgen receptor (AR) is a major factor responsible for the gender disparity observed in HCC $(7,8)$. The AR, also known as the nuclear receptor subfamily 3 , group C, member 4 , is a $90-\mathrm{kDa}$ ligand-dependent transcription factor, which regulates the expression of target genes. As it belongs to the nuclear receptor superfamily, the AR gene contains three primary functional domains: i) the $\mathrm{N}$-terminal transactivation domain; ii) the DNA-binding domain; and iii) the androgen-binding domain (9). The main function of the AR is to mediate the effects of androgens in important pathological and physiological processes, including the formation and evolution of cancer $(10,11)$. Following androgen-mediated activation, the AR translocates to the nucleus and binds to the androgen response element (ARE) of its target genes in order to activate their expression $(9,10)$. In certain conditions, the AR has been identified to be activated by non-androgenic pathways. Furthermore, previous studies have reported that AR can be activated by a number of other molecules, including interleukin-6, $\beta$-catenin and acetylcholine (ACh) (12-14).

The expression of AR has been investigated in HCC cell lines and tissues (15-17). Previous studies have demonstrated that the AR protein and mRNA expression levels were $>1.5$-fold higher in HCC tissues, and that AR overexpression was associated with the incidence of $\operatorname{HCC}(15,17,18)$. In addition, the results of AR function analyses in mouse models have suggested that AR overexpression promotes tumorigenicity $(19,20)$. By contrast, the suppression of AR by small interfering RNA has been identified to induce cell cycle arrest at the $\mathrm{G}_{1}$ phase and inhibit $\mathrm{HCC}$ cell growth (18). In addition, previous data have indicated that the $\mathrm{AR}$ is able to modulate the expression and activity of a number of molecules within HCC cells $(18,21,22)$. These molecules are associated with cellular proliferation, tumor growth and/or metastasis. Notably, studies on transgenic mouse models have demonstrated that 
Table I. Overexpression of androgen receptor in human tumors.

\begin{tabular}{lccc}
\hline First author (Ref.) & Cases investigated, $\mathrm{n}$ & Cases with elevated AR, $\mathrm{n}$ & Year \\
\hline Feng et al (18) & 33 & 19 & 2011 \\
Chen et al (25) & 20 & 16 & 2012 \\
Ma et al $(22)$ & 10 & 7 & 2008 \\
Barone et al (15) & 20 & 20 & 2009 \\
\hline
\end{tabular}

AR may play a negative role in HCC metastasis (22). The aforementioned findings indicate that AR exhibits marked oncogenic properties in HCC initiation and development in the early stages of the disease, but functions as a suppressor of metastasis in late-stage HCC. Targeting AR in order to treat $\mathrm{HCC}$ at different stages may represent an improved therapeutic approach compared with androgen ablation therapy. The present study reviewed the available literature regarding the significance of AR in hepatocarcinogenesis.

\section{AR alterations in $\mathrm{HCC}$}

Significant efforts have been made in order to investigate the expression of the AR in HCC tissue specimens and cell lines $(16,23,24)$. In early studies, the percentage positivity for AR expression in tissue specimens was found to vary significantly. These variations may have been the result of using indirect binding assays, which were not sufficiently sensitive to detect AR expression (16). Table I summarizes the findings of recent studies that used human tissue samples.

AR is expressed in a variety of HCC cell lines, including THLE-2, HepG2, Huh7, SNU-387 and LM-3 (18,21). A higher AR expression has been detected in cells that exhibit a larger number of malignant features (21). The difference in AR expression between HCC cells with high and low metastatic potential suggests that a high expression of AR may contribute to HCC cell invasion and migration (21). In previous studies that also analyzed control tissues, a higher AR expression was evident in tumoral tissues compared with peritumoral tissues, revealing that the disruption in AR homeostasis was associated with the incidence of HCC $(15,18)$. Overexpression of AR in HCC cells may lead to the dysregulation of several molecules associated with cellular proliferation, tumor growth and/or metastasis $(21,22,25)$. Findings from clinical $\mathrm{HCC}$ specimens revealed that an elevation in the levels of microRNA (miR)-216a and cell cycle-related kinase (CCRK) was positively correlated with an increase in the AR protein level, and that the overexpression of miR-216a and CCRK was associated with poor patient prognoses $(18,25)$. By contrast, Ma et al (22) established that AR overexpression was only present in tumors with a size of $<3 \mathrm{~cm}$ (22). In addition, these authors identified a low expression of AR in severe HCC lesions (22). These results appear to be in agreement with those of Zhu et al (26), who demonstrated that the expression of AR was higher in peritumoral tissues compared with tumor tissues (26). Due to these conflicting results regarding the expression of AR in HCCs, a detailed investigation into the association between AR expression and tumor grade during the progression of $\mathrm{HCC}$ is required.
At present, the mechanisms underlying AR-induced hepatocarcinogenesis remain to be elucidated. In the following sections, recent advances in molecular research that have been conducted in order to understand the role of AR in hepatocarcinogenesis are reviewed. The roles of AR are also shown in Fig. 1.

\section{AR promotes hepatocarcinogenesis}

$A R$ and the Wnt signaling pathway. HCC is a heterogeneous cancer involving the aberrant activation of Wnt signaling (27). $\beta$-Catenin is an important effector molecule in the Wnt signaling pathway (28). An abnormal regulation of $\beta$-catenin has been described in up to $90 \%$ of HCC cases, which has been identified as a major early carcinogenic event that occurs during the development of HCC (29). The crosstalk between AR and Wnt/ $\beta$-catenin signaling has been established by previous genetic studies $(18,30,31)$.

Feng et al (18) revealed that AR may be able to activate $\beta$-catenin during heptocarcinogenesis by modulating CCRK. CCRK is a novel cyclin-dependent kinase-activating kinase that serves as an important regulator of tumorigenicity in a number of human malignancies (32). Ligand-activated AR has been demonstrated to stimulate the transcription of CCRK in human liver and HCC cells, while the presence of ARE was identified in the CCRK promoter region. In addition, the AR-induced CCRK was demonstrated to activate $\beta$-catenin signaling, which is known to be associated with glycogen synthase kinase $3 \beta$ phosphorylation. RNA interference-mediated ablation of CCRK or $\beta$-catenin resulted in a significant suppression of $A R$-induced $G_{1} / S$ cell cycle progression. Furthermore, the aberrant expression of CCRK in HCC cells led to an increase in the levels of AR, whilst the downregulation of $\beta$-catenin attenuated the effects of CCRK on AR. This suggests that a cycle exists within HCC cells: AR stimulates CCRK expression in order to induce the activity of $\beta$-catenin, whilst $\beta$-catenin acting downstream of CCRK induces the expression and activity of AR. An upregulation in the expression levels of AR, CCRK and active $\beta$-catenin was observed in primary $\mathrm{HCC}$ specimens, which further supports the role of the AR/CCRK/ $\beta$-catenin-positive regulatory circuit in hepatocarcinogenesis (18).

$A R$ and miR-216a. miRNAs are small, non-coding RNA molecules, which inhibit the expression levels of target genes. Previous studies have suggested that miRNAs may be another group of host genetic factors that are associated with hepatocarcinogenesis $(33,34)$. Increasing evidence demonstrated that dysregulated miRNA expression is commonly 


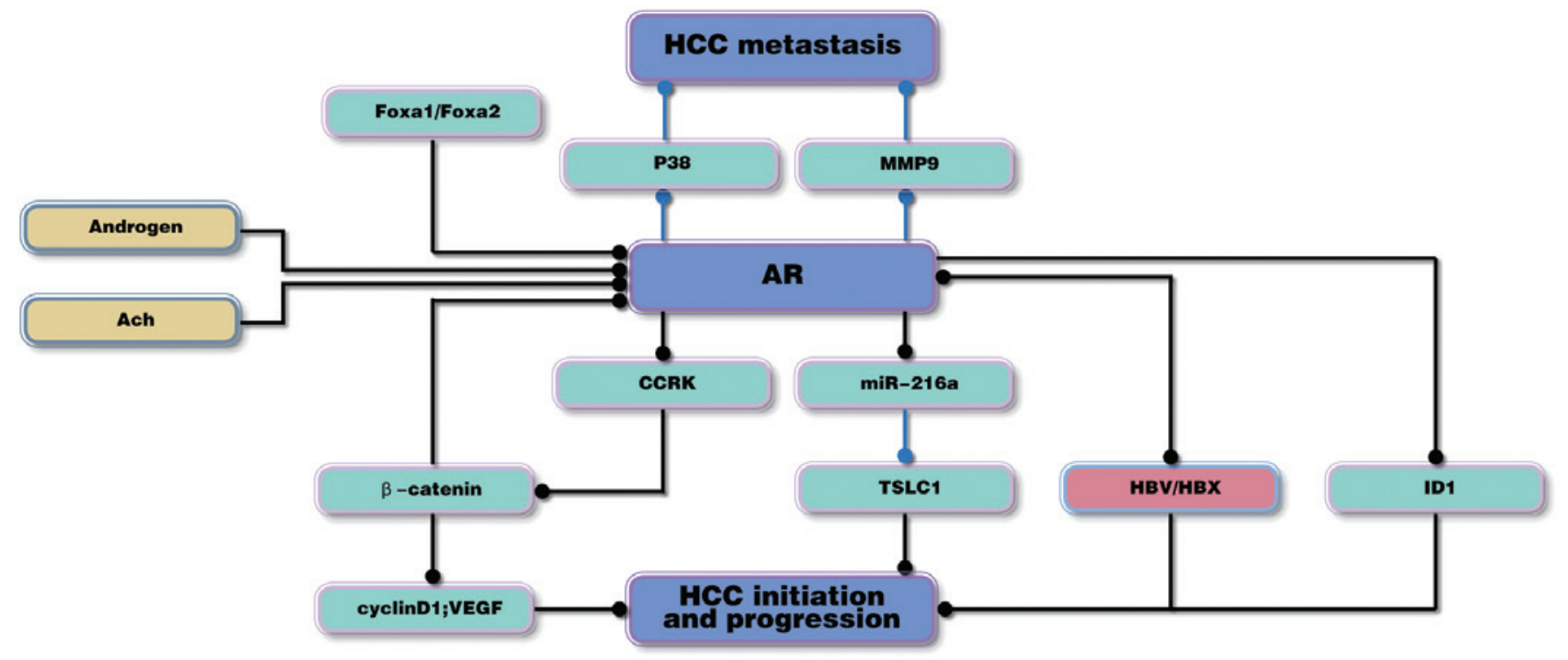

Stimulatory modification

Inhibitory modifciation

Figure 1. AR may have a dual role, promoting HCC initiation in the early stages, but suppressing HCC metastasis in the advanced stages of the disease. AR, androgen receptor; HCC, hepatocellular carcinoma; ACh, acetylcholine; Fox, forkhead box; MMP9, matrix metalloproteinase 9; CCRK, cell cycle-related kinase; miR-216a, microRNA-216a; TSLC1, tumor suppressor in lung cancer 1; HBV, hepatitis B virus; HBX, HBV X protein; ID1, inhibitor of differentiation 1; VEGF, vascular endothelial growth factor.

observed in HCCs (35). However, the mechanism through which a disturbance in cellular miRNAs affects HCC initiation and progression remains to be elucidated. A study by Chen et al (25) identified that ligand-stimulated AR was a regulator of miR-216a and lead to enhanced tumorigenesis. Furthermore, these authors revealed that the level of miR-216a was significantly higher in male HCC patients (25). This male-predominant increase in the expression of miR-216a suggests that the crosstalk between AR and miR-216a may be involved in hepatocarcinogenesis. In vivo studies have identified that the ligand-stimulated AR can bind to ARE site residues within the promoter regions and stimulate the transcription of primary miR-216a, resulting in an increase in the expression of miR-216a (25). This increase stimulates the proliferation, migration and invasion of liver cancer cells through a decrease in the expression of tumor suppressor in lung cancer 1 (TSLC1), a protein associated with tumor invasion and metastasis $(36,37)$. These results are in agreement with the finding that AR and miR-216a were concordantly overexpressed in clinical specimens, while an inverse correlation between TSLC1 and miR-216a was also identified in these liver tissues (25). The presence of the hepatitis B virus (HBV) $\mathrm{X}$ protein (HBx) can further augment the effect of AR-mediated miR-216a transcription, due to its known ability to enhance AR activity $(38,39)$. Notably, recent studies have indicated that miRNAs, including miR-224, miR-221, miR-122a and miR-223, demonstrate deregulated expression patterns in male HCC patients (25). Therefore, the dysregulation in the expression of these miRNAs is hypothesized to be, at least in part, mediated by AR. Further investigation is required in order to validate this hypothesis.
$A R$ and $H B V . \mathrm{HBV}$ is a hepatotropic virus, which chronically infects $\sim 350$ million individuals worldwide (40). Increasing evidence has established a clear association between persistent HBV infection and the development of HCC (41-43). Previous studies have revealed that the long-term risk of developing HCC was markedly increased in patients with higher serum HBV DNA concentrations. Furthermore, antiviral therapies have demonstrated a reduction in the occurrence of HCC following a decrease in the serum levels of HBV DNA (44). Using mice transfected with the entire HBV genome, which productively produced HBV in their liver, Tian et al (45) demonstrated that HBV replicated $\sim 2$ times more efficiently in male mice compared with female mice. The higher replication efficiency of HBV detected in the male mice provided an explanation for the gender discrepancy observed in cases of HCC. Further studies have indicated that androgen and AR are important for HBV replication (42). In total, two AREs exist within the HBV genome. The castration of male mice or the introduction of mutagenesis to remove these two AREs results in a reduction in the level of HBV surface antigen, DNA and RNA. This observation confirms the importance of androgen and $\mathrm{AR}$ in the mediation of $\mathrm{HBV}$ replication.

A study by Wu et al (46) indicated that an aberrant overexpression of AR in HCC cells cooperates with HBV to enhance cell growth and invasion in vitro, and initiate $\mathrm{HCC}$ in vivo. Further studies have demonstrated that $\mathrm{HBx}$, a HBV-encoded protein required for efficient viral replication, enhanced the gene transactivation activity of AR in the presence of androgens $(38,39)$. These data suggest that AR induces a positive feedback response upon HBV production, in order to mediate hepatocarcinogenesis as follows: i) The activated 
AR increases HBV replication by binding to the ARE within the HBV genome; ii) the increased level of HBV RNA leads to the production of more HBV viral antigen and DNA; iii) the upregulated expression of $\mathrm{HBx}$ following AR-enhanced HBV RNA transcription ultimately promotes AR transactivation; and iv) the increased viral antigens then cooperate with AR to increase the process of hepatocarcinogenesis.

$A R$ and forkhead box (FOX)A1/2. FOX proteins are a family of transcription factors, which regulate the expression of target genes involved in cellular differentiation, growth and proliferation (47). The family consists of the FOXA1, FOXA2, and FOXA3 proteins. Previous studies using FOXA-null mice demonstrated the combined importance of FOXA1 and FOXA2 in liver cell specification and differentiation, and identified a potential role for FOXA1/2 in human liver diseases (48-50).

A recent study investigated the association between FOXA1/2 and AR transactivation (51). The results demonstrated that a FOXA1/2 deficiency significantly reduced the tumor size in male mice following the administration of $\mathrm{N}$-nitrosodiethylamine, which suggests that FOXA1/2 is required in order for AR to promote tumor growth. Genomic distribution analysis revealed that the majority of AR-associated genes were also bound by FOXA1 or FOXA2. The FOXA1/2 and AR binding sites were located close together at the regulatory regions of the common target genes. A functional annotation of the FOXA/AR dual target genes identified that AR and FOXA1/2 promote tumor growth via a number of pathways, including the cell cycle, DNA replication and cell growth and proliferation. These results indicated that FOXA1/2 and AR are involved in the control of gene expression in $\mathrm{HCC}$ and are involved in the promotion of HCC. This finding is further supported by the observation that the co-occupancy of FOXA1/2 and AR markedly increased in the livers of male mice following carcinogen treatment. By contrast, mutation of FOXA1/2 prevented AR from binding to the common FOXA/AR targets and attenuated the AR-induced proliferation and malignant transformation of cells (51).

AR and inhibitor of differentiation 1 (IDI). Previous genetic studies have revealed that the ID1 gene is a potential downstream target of AR in HCC cells (21). ID1 belongs to the basic helix-loop helix family of genes, which have been detected in multiple malignant tumors. In addition, the expression levels of these genes have been associated with invasive features of cancer $(52,53)$. Increasing evidence has indicated that ID1 is upregulated in HCC samples and induces cellular proliferation. Overexpression of ID1 has been identified as a marker of unfavorable prognoses in HCC patients $(54,55)$. A study by Ao et al (21) demonstrated that the androgen agonist, R1881, increased the migration and invasion of AR-positive HCC cells through the stimulation of ID1. By contrast, the treatment of AR-negative HCC cells with R1881 resulted in no change in the mRNA and protein levels of ID1. These findings suggest that activated AR is important for the induction of ID1. Furthermore, a depletion in ID1 levels has been revealed to attenuate AR-induced cell migration and invasion, which indicates that ID1 is a major mediator of AR-induced cell migration and invasion. However, the mechanism underlying ID1 expression induced by activated AR remains to be elucidated. Chromatin immunoprecipitation assays have revealed that AR does not bind directly to the ID1 promoter, which suggests that the increased in ID1 expression that is induced by AR may be a secondary response (21). Further investigation is required in order to determine the molecular mechanisms underlying AR-induced ID1 expression.

Non-androgen-mediated AR signals in hepatocarcinogenesis. A previous study reported that AR-positive HCC cells are able to grow in the absence of androgen, and that the addition of dihydrotestosterone (DHT) had little effect on AR-induced cell growth (20). Another study, which stably transfected functional AR into AR-negative HCC cells, revealed that cell growth was increased in the absence of androgen (46). These observations indicate that non-androgen-mediated AR signals may also be involved in hepatocarcinogenesis.

Increasing evidence suggests that $\mathrm{HBx}$ is able to enhance AR activity and increase AR-mediated cell transformation in the presence and absence of DHT $(39,46)$, which may explain the higher incidence of male HBV-associated HCC (38). Nie et al (14) revealed that $\mathrm{ACh}$ promotes the migration and invasion of HCC cells by activating the AR transcriptional activity and upregulating AR expression. Previous studies have demonstrated that the ACh degradation enzyme, acetylcholinesterase, is downregulated in HCC. This can lead to an increase in the level of $\mathrm{ACh}$, which subsequently activates the $\mathrm{ACh}$ receptor $(\mathrm{AChR})$ and promotes the proliferation of HCC cells (56). In addition, Nie et al (14) demonstrated that the expression of AR was upregulated by $\mathrm{ACh}$ in a dose-dependent manner in HCC cells, and that the upregulated expression of AR was inhibited by the AChR antagonist, mecamylamine. Furthermore, downregulation of AR was identified to significantly reverse the ACh-induced migration and invasion of HCC cells, which indicates that AR is a major mediator of ACh-induced tumorigenicity. Considering the role of non-androgen-mediated AR signals in hepatocarcinogenesis and the observation that androgen ablation therapy in treating HCC provided inconsistent results $(57,58)$, it may be hypothesized that the AR, rather than androgens, represent an improved therapeutic approach to target HCC.

\section{AR suppresses HCC metastasis}

AR is known to be involved in the promotion of the genesis and evolution of HCC. However, a previous study revealed that AR may function as a suppressor of metastasis in advanced stages of HCC (22). Using an AR-knockout mouse model with carcinogen-induced HCC, Ma et al (22) demonstrated that mice lacking hepatic AR developed HCC later, but exhibited a more severe malignant phenotype with a higher risk of lung metastasis and succumbed to the disease earlier. Further biochemical studies indicated that AR may suppress HCC metastasis via multiple signaling pathways (22).

p38 kinases are a class of mitogen-activated protein kinases, which convert extracellular stimuli into a number of cellular responses, including survival, proliferation, migration and differentiation (52). Deregulation of p38 has been identified to be involved in hepatocarcinogenesis $(59,60)$. Ma et al (22) revealed that a higher AR expression may enhance cell anoikis by decreasing the phosphorylation and inhibiting the 
activity of $\mathrm{p} 38$, thereby suppressing metastasis. However, the mechanism underlying p38 dephosphorylation induced by AR remains to be elucidated. In addition to regulating $\mathrm{HCC}$ invasion by modulating p38 phosphorylation, AR has been identified to regulate the expression of matrix metalloproteinase (MMP)9. MMP9 is the founding member of the MMP family, which is comprised of $\geq 40$ proteins (61). There is clear evidence to indicate that the expression of MMP9 is higher in cases of HCC with metastasis $(62,63)$. In addition, molecular analysis has revealed that AR downregulates MMP9 expression through the inhibition of nuclear factor- $\kappa \mathrm{B}$ signaling (22). These effects of AR on HCC metastasis suggest that AR is involved in a wide range of cellular signaling pathways, and has dual roles at different disease stages.

\section{AR as a promising target for cancer therapy}

A number of clinical trials that used various anti-androgens to treat HCC have yielded poor results $(57,58)$. For instance, a small-scale phase II clinical trial using flutamide failed to establish any evidence supporting improved survival. Similar results were also observed in large-scale population studies using leuprorelin and flutamide $(57,64)$. Several hypotheses exist that may be able to explain these controversial results observed in clinical trials and basic studies. The current literature suggests that androgen-mediated and non-androgen-mediated AR signals are involved in hepatocarcinogenesis, which indicates that the expression of AR affects tumor growth, whereas the androgen concentration does not have a similar effect $(14,20,46)$. The anti-androgens used in clinical trials were developed in order to compete with androgens for binding to AR. In addition, increasing evidence suggests that $\mathrm{AR}$ is a negative regulator of metastasis in advanced stages of HCC (22). Yet the majority of anti-androgen based trials included patients with advanced stage and unresectable HCCs; therefore, anti-androgens are likely to be less effective in such cases. On the basis of the aforementioned evidence, it is conceivable to hypothesize that $\mathrm{AR}$ may be a promising candidate for molecular-targeted HCC therapy. Targeting the AR should be stage-dependent by blocking the action of AR during the early stages and upregulating the expression of AR at the metastatic stage of the disease.

\section{Conclusions}

Following the identification of the critical role of AR signaling in hepatocarcinogenesis and the involvement of additional nuclear hormone receptor signals, HCC should be considered as an endocrine-associated neoplasm $(18,20)$. Although the specific mechanisms underlying AR regulation in $\mathrm{HCC}$ remain to be elucidated, various findings can be merged in order to provide a perspective of the oncogenic properties of $A R$ in HCC (18,20-22). Androgenic and non-androgenic pathways have been demonstrated to activate the AR during hepatocarcinogenesis $(14,18,46)$. AR exhibits marked oncogenic properties in the early stages of HCC development, but may suppress HCC metastasis in the advanced stages of HCC (22). Several upstream and downstream targets of AR involved in mediating cellular proliferation and transformation have been identified in HCC $(14,21,22,25)$. The primary objective of these studies was to translate these findings into clinical applications. The available results support the hypothesis that targeting the AR, rather than the androgen, may provide an improved therapeutic approach for the treatment of HCC.

\section{References}

1. Yang JD and Roberts LR: Hepatocellular carcinoma: A global view. Nat Rev Gastroenterol Hepatol 7: 448-458, 2010.

2. El-Serag HB: Epidemiology of viral hepatitis and hepatocellular carcinoma. Gastroenterology 142: 1264-1273, 2012.

3. Ascha MS, Hanouneh IA, Lopez R, Tamimi TA, Feldstein AF and Zein NN: The incidence and risk factors of hepatocellular carcinoma in patients with nonalcoholic steatohepatitis. Hepatology 51: 1972-1978, 2010.

4. Rahbari NN, Mehrabi A, Mollberg NM, et al: Hepatocellular carcinoma: current management and perspectives for the future. Ann Surg 253: 453-469, 2011.

5. Altekruse SF, McGlynn KA and Reichman ME: Hepatocellular carcinoma incidence, mortality and survival trends in the United States from 1975 to 2005. J Clin Oncol 27: 1485-1491, 2009.

6. Bruix J and Sherman M; American Association for the Study of Liver Diseases: Management of hepatocellular carcinoma: an update. Hepatology 53: 1020-1022, 2011.

7. Yeh SH and Chen PJ: Gender disparity of hepatocellular carcinoma: the roles of sex hormones. Oncology 78 (Suppl 1): 172-179, 2010.

8. Ruggieri A, Barbati C and Malorni W: Cellular and molecular mechanisms involved in hepatocellular carcinoma gender disparity. Int J Cancer 127: 499-504, 2010.

9. Gelmann EP: Molecular biology of the androgen receptor. J Clin Oncol 20: 3001-3015, 2002.

10. Simental JA, Sar M, Lane MV, French FS and Wilson EM: Transcriptional activation and nuclear targeting signals of the human androgen receptor. J Biol Chem 266: 510-518, 1991.

11. Zegarra-Moro OL, Schmidt LJ, Huang H and Tindall DJ: Disruption of androgen receptor function inhibits proliferation of androgen-refractory prostate cancer cells. Cancer Res 62: 1008-1013, 2002.

12. Truica CI, Byers S and Gelmann EP: Beta-catenin affects androgen receptor transcriptional activity and ligand specificity. Cancer Res 60: 4709-4713, 2000.

13. Yeh S, Lin HK, Kang HY, Thin TH, Lin MF and Chang C: From HER2/Neu signal cascade to androgen receptor and its coactivators: a novel pathway by induction of androgen target genes through MAP kinase in prostate cancer cells. Proc Natl Acad Sci USA 96: 5458-5463, 1999.

14. Nie H, Cao Q, Zhu L, Gong Y, Gu J and He Z: Acetylcholine acts on androgen receptor to promote the migration and invasion but inhibit the apoptosis of human hepatocarcinoma. PLoS One 8: e61678, 2013.

15. Barone M, Margiotta M, Scavo MP, et al: Possible involvement of androgen receptor alterations in hepatocarcinogenesis. Dig Liver Dis 41: 665-670, 2009.

16. Kalra M, Mayes J, Assefa S, Kaul AK and Kaul R: Role of sex steroid receptors in pathobiology of hepatocellular carcinoma. World J Gastroenterol 14: 5945-5961, 2008.

17. Villa E: Androgen receptor alterations in hepatocarcinogenesis. Dig Liver Dis 41: 632-633, 2009.

18. Feng H, Cheng AS, Tsang DP, et al: Cell cycle-related kinase is a direct androgen receptor-regulated gene that drives $\beta$-catenin/T cell factor-dependent hepatocarcinogenesis. J Clin Invest 121: 3159-3175, 2011.

19. Zender L and Kubicka S: Androgen receptor and hepatocarcinogenesis: what do we learn from HCC mouse models? Gastroenterology 135: 738-740, 2008.

20. Ma WL, Hsu CL, Wu MH, et al: Androgen receptor is a new potential therapeutic target for the treatment of hepatocellular carcinoma. Gastroenterology 135: 947-955, 2008.

21. Ao J, Meng J, Zhu L, et al: Activation of androgen receptor induces ID1 and promotes hepatocellular carcinoma cell migration and invasion. Mol Oncol 6: 507-515, 2012.

22. Ma WL, Hsu CL, Yeh CC, et al: Hepatic androgen receptor suppresses hepatocellular carcinoma metastasis through modulation of cell migration and anoikis. Hepatology 56: 176-185, 2012.

23. Vizoso FJ, Rodriguez M, Altadill A, et al: Liver expression of steroid hormones and Apolipoprotein D receptors in hepatocellular carcinoma. World J Gastroenterol 13: 3221-3227, 2007. 
24. Tavian D, De Petro G, Pitozzi A, Portolani N, Giulini SM and Barlati S: Androgen receptor mRNA under-expression in poorly differentiated human hepatocellular carcinoma. Histol Histopathol 17: 1113-1119, 2002.

25. Chen PJ, Yeh SH, Liu WH, et al: Androgen pathway stimulates microRNA-216a transcription to suppress the tumor suppressor in lung cancer-1 gene in early hepatocarcinogenesis. Hepatology 56 : 632-643, 2012.

26. Zhu R, Zhang JS, Zhu YZ, et al: HBx-induced androgen receptor expression in $\mathrm{HBV}$-associated hepatocarcinoma is independent of the methylation status of its promoter. Histol Histopathol 26 : 23-35, 2011.

27. Dahmani R, Just PA and Perret C: The Wnt/ß-catenin pathway as a therapeutic target in human hepatocellular carcinoma. Clin Res Hepatol Gastroenterol 35: 709-713, 2011.

28. Lee JM, Yang J, Newell P, et al: $\beta$-Catenin signaling in hepatocellular cancer: Implications in inflammation, fibrosis, and proliferation. Cancer Lett 343: 90-97, 2014.

29. Nault JC and Zucman-Rossi J: Genetics of hepatobiliary carcinogenesis. Semin Liver Dis 31: 173-187, 2011

30. Lee E, Madar A, David G, Garabedian MJ, Dasgupta R and Logan SK: Inhibition of androgen receptor and $\beta$-catenin activity in prostate cancer. Proc Natl Acad Sci USA 110: 15710-15715, 2013.

31. Li Y, Zheng Y, Izumi K, et al: Androgen activates $\beta$-catenin signaling in bladder cancer cells. Endocr Relat Cancer 20 293-304, 2013.

32. Tian Y, Wan H and Tan G: Cell cycle-related kinase in carcinogenesis. Oncol Lett 4: 601-606, 2012.

33. Takahashi K, Yan I, Wen HJ and Patel T: microRNAs in liver disease: from diagnostics to therapeutics. Clin Biochem 46 : 946-952, 2013.

34. Gailhouste L and Ochiya T: Cancer-related microRNAs and their role as tumor suppressors and oncogenes in hepatocellular carcinoma. Histol Histopathol 28: 437-451, 2013.

35. Guo J and Friedman SL: The expression patterns and clinical significance of microRNAs in liver diseases and hepatocellular carcinoma. Curr Pharm Des 19: 1262-1272, 2013.

36. Liang QL, Chen GQ, Li ZY and Wang BR: Function and histopathology of a cell adhesion molecule TSLC1 in cancer. Cancer Invest 29: 107-112, 2011.

37. Zhang J, Ning J, Geng J, Cui B and Dong X: Down-regulation of tumor suppressor in lung cancer 1 (TSLC1) expression correlates with poor prognosis in patients with colon cancer. J Mol Histol 43: 715-721, 2012.

38. Chiu CM, Yeh SH, Chen PJ, et al: Hepatitis B virus X protein enhances androgen receptor-responsive gene expression depending on androgen level. Proc Natl Acad Sci USA 104: 2571-2578, 2007.

39. Zheng Y, Chen WL, Ma WL, Chang C and Ou JH: Enhancement of gene transactivation activity of androgen receptor by hepatitis B virus X protein. Virology 363: 454-461, 2007.

40. Lok AS and McMahon BJ: Chronic hepatitis B: update 2009. Hepatology 50: 661-662, 2009.

41. Xu C, Zhou W, Wang Y and Qiao L: Hepatitis B virus-induced hepatocellular carcinoma. Cancer Lett 345: 216-222, 2014.

42. Bharadwaj M, Roy G, Dutta K, Misbah M, Husain M and Hussain S: Tackling hepatitis B virus-associated hepatocellular carcinoma - the future is now. Cancer Metastasis Rev 32: 229-268, 2013

43. Pollicino T, Saitta C and Raimondo G: Hepatocellular carcinoma: the point of view of the hepatitis B virus. Carcinogenesis 32 1122-1132, 2011

44. Giacomin A, Cazzagon N, Sergio A, Vanin V and Farinati F: Hepatitis B virus-related hepatocellular carcinoma: primary, secondary, and tertiary prevention. Eur J Cancer Prev 20: 381-388, 2011.

45. Tian Y, Kuo CF, Chen WL and Ou JH: Enhancement of hepatitis B virus replication by androgen and its receptor in mice. J Virol 86: 1904-1910, 2012.
46. Wu MH, Ma WL, Hsu CL, et al: Androgen receptor promotes hepatitis B virus-induced hepatocarcinogenesis through modulation of hepatitis B virus RNA transcription. Sci Transl Med 2: 32ra35, 2010

47. Xia L, Huang W, Tian D, et al: Forkhead box Q1 promotes hepatocellular carcinoma metastasis by transactivating ZEB2 and VersicanV1 expression. Hepatology 59: 958-973, 2014.

48. Xia L, Huang W, Tian D, et al: ACP5, a direct transcriptional target of FoxM1, promotes tumor metastasis and indicates poor prognosis in hepatocellular carcinoma. Oncogene 33: 1395-1406, 2014

49. Liang C, Chen W, Zhi X, et al: Serotonin promotes the proliferation of serum-deprived hepatocellular carcinoma cells via upregulation of FOXO3a. Mol Cancer 12: 14, 2013.

50. Wang W, He S, Ji J, Huang J, Zhang S and Zhang Y: The prognostic significance of FOXQ1 oncogene overexpression in human hepatocellular carcinoma. Pathol Res Pract 209: 353-358, 2013.

51. Li Z, Tuteja G, Schug J and Kaestner KH: Foxa1 and Foxa2 are essential for sexual dimorphism in liver cancer. Cell 148: 72-83, 2012.

52. Su Y, Gao L, Teng L, et al: Id1 enhances human ovarian cancer endothelial progenitor cell angiogenesis via PI3K/Akt and NF-кB/MMP-2 signaling pathways. J Transl Med 11: 132, 2013.

53. Guo Q, Guo P, Mao Q, et al: ID1 affects the efficacy of radiotherapy in glioblastoma through inhibition of DNA repair pathways. Med Oncol 30: 325, 2013

54. Ding R, Han S, Lu Y, et al: Overexpressed Id-1 is associated with patient prognosis and $\mathrm{HBx}$ expression in hepatitis B virus-related hepatocellular carcinoma. Cancer Biol Ther 10: 299-307, 2010.

55. Damdinsuren B, Nagano H, Kondo M, et al: Expression of Id proteins in human hepatocellular carcinoma: relevance to tumor dedifferentiation. Int J Oncol 26: 319-327, 2005.

56. Zhao Y, Wang X, Wang T, et al: Acetylcholinesterase, a key prognostic predictor for hepatocellular carcinoma, suppresses cell growth and induces chemosensitization. Hepatology 53: 493-503, 2011.

57. Groupe d'Etude et de Traitement du Carcinome Hépatocellulaire: Randomized trial of leuprorelin and flutamide in male patients with hepatocellular carcinoma treated with tamoxifen. Hepatology 40: 1361-1369, 2004.

58. Forbes A, Wilkinson ML, Iqbal MJ, Johnson PJ and Williams R: Response to cyproterone acetate treatment in primary hepatocellular carcinoma is related to fall in free 5 alpha-dihydrotestosterone. Eur J Cancer Clin Oncol 23: 1659-1664, 1987.

59. Iyoda K, Sasaki Y, Horimoto M, et al: Involvement of the p38 mitogen-activated protein kinase cascade in hepatocellular carcinoma. Cancer 97: 3017-3026, 2003.

60. Koul HK, Pal M and Koul S: Role of p38 MAP kinase signal transduction in solid tumors. Genes Cancer 4: 342-359, 2013.

61. Zhang J, Zhang D, Wu GQ, Feng ZY and Zhu SM: Propofol inhibits the adhesion of hepatocellular carcinoma cells by upregulating microRNA-199a and downregulating MMP-9 expression. Hepatobiliary Pancreat Dis Int 12: 305-309, 2013.

62. Gao J, Ding F, Liu Q and Yao Y: Knockdown of MACC1 expression suppressed hepatocellular carcinoma cell migration and invasion and inhibited expression of MMP2 and MMP9. Mol Cell Biochem 376: 21-32, 2013.

63. Zhang M, Dai C, Zhu H, et al: Cyclophilin A promotes human hepatocellular carcinoma cell metastasis via regulation of MMP3 and MMP9. Mol Cell Biochem 357: 387-395, 2011

64. Chao Y, Chan WK, Huang YS, et al: Phase II study of flutamide in the treatment of hepatocellular carcinoma. Cancer 77: 635-639, 1996 